\title{
Longitudinal Trends in Fall Accidents in Community Dwelling Korean Adults:
}

\section{The 2008-2013 Korean Community Health Survey}

\author{
Ickpyo Hong, PhD, OTR/L ${ }^{1}$, Annie N. Simpson, $\mathrm{PhD}^{2}$, Sarah Logan, $\mathrm{PhD}^{2}$, Hee-Soon Woo, $\mathrm{PhD}^{3}$
}

\begin{abstract}
${ }^{1}$ Department of Health Sciences and Research, College of Health Professions, Medical University of South Carolina, Charleston, SC, USA; ${ }^{2}$ Department of Healthcare Leadership and Management, College of Health Professions, Medical University of South Carolina, Charleston, SC, USA; ${ }^{3}$ Department of Occupational Therapy, Wonkwang University School of Medicine, Iksan, Korea
\end{abstract}

\begin{abstract}
Objective To describe the longitudinal characteristics of unintentional fall accidents using a representative population-based sample of Korean adults.

Methods We examined data from the Korean Community Health Survey from 2008 to 2013. Univariate analysis and multivariable logistic regression were used to identify the characteristics of fall accidents in adults.

Results Between 2008 and 2013, the incidence rate of fall accidents requiring medical treatment increased from 1,248 to 3,423 per 100,000 people $(\mathrm{p}<0.001)$, while the proportion of indoor fall accidents decreased from $38.12 \%$ to $23.16 \%(p<0.001)$. Females had more annual fall accidents than males $(\mathrm{p}<0.001)$. The major reason for fall accidents was slippery floors (33.7\% in 2011 and 36.3\% in 2013). Between 2008 and 2010, variables associated with higher fall accident risk included specific months (August and September), old age, female gender, current drinker, current smoker, diabetes, osteoarthritis, osteoporosis, and depression. A high level of education and living with a partner were negatively associated with fall accident risk. In 2013, people experiencing more than 1 fall accident felt more fear of falling than those having no fall accidents (odds ratio [OR] for 1 fall, 2.12; 95\% confidence interval [CI], 2.04-2.12; OR for more than 2 falls, 2.97; 95\% CI, 2.83-3.10).

Conclusion The occurrence of fall accidents has consistently increased in Korea from 2008 to 2013. Future intervention studies are needed to reduce the increasing incidence rates of fall accidents in community dwelling adults.
\end{abstract}

Keywords Accidental falls, Life style, Fear, Adult, Korean

Received July 2, 2015; Accepted October 27, 2015

Corresponding author: Ickpyo Hong

Department of Health Sciences and Research, College of Health Professions, Medical University of South Carolina, 151-B Rutledge Avenue, MSC 962, Room B417, Charleston, SC 29425, USA. Tel: +1-412-925-2224, Fax: +1-843-792-0710, E-mail: hongi@musc.edu

ORCID: Ickpyo Hong (http://orcid.org/0000-0001-5404-7646); Annie N. Simpson (http://orcid.org/0000-0002-7216-1036); Hee-Soon Woo (http:// orcid.org/0000-0003-4712-9808).

(c) This is an open-access article distributed under the terms of the Creative Commons Attribution Non-Commercial License (http://creativecommons.org/ licenses/by-nc/4.0) which permits unrestricted noncommercial use, distribution, and reproduction in any medium, provided the original work is properly cited. Copyright $\odot 2016$ by Korean Academy of Rehabilitation Medicine 


\section{INTRODUCTION}

In Korea, the cause-specific death rate of fall accidents was 4.6 per 100,000 people in 2013 , a rate even higher than for breast cancer, sepsis, and tuberculosis $(4.4,4.3$, and 4.1) [1]. As falls are the third highest non-disease cause of death in Korea, it is critical to have a better understanding of fall accident trends. Since 2008, the Korean Centers for Disease Control and Prevention (KCDC) have conducted an annual national health survey, the Korean Community Health Survey (KCHS), which covers a wide variety of health topics, including extensive information regarding falls [2].

Unfortunately, although the nationwide survey data has been available to the public, most Korean research papers cite foreign studies to describe the incidence of falls in Korea [3-5], and the information about falls from the cited Western studies is often outdated [6,7]. Several studies have reported incidence rates in Korea; however, the results were not consistent due to varying sample size, small resident area, a specific target population, and differing methodological approaches [3-5,8]. For example, an observational study randomly recruited 335 community-dwelling older adults from a cohort study and reported that $15 \%$ of their sample had experienced falls. In contrast, a prospective study reported a high fall occurrence of $41.6 \%$ when they conducted personal interviews in a small city with 260 community dwelling older adults [4].

Since 2008, the KCDC has annually reported summary statistics of the KCHS, including falls [9,10]. In addition, a recent study used the 2011 KCHS to report the prevalence of falls [11]. However, the minimally descriptive report is limited to single annual fall information only $[10,11]$. It is unclear whether the trends of falls among community dwelling adults have been consistent or varied dramatically in consecutive years in Korea, because few studies have been conducted using national data to examine falls over extended time periods.

An investigation of a series of national level datasets may provide important information about the general trends in fall accidents in Korea. The general trends will outline the change in relationships between fall accidents and demographic characteristics and environmental factors for consecutive years. Therefore, the aim of this study is to investigate the incidence rates of fall accidents and the characteristics of people who experienced falls during a 7-year period (2008-2013) in South Korea.

\section{MATERIALS AND METHODS}

\section{Database and study population}

This study utilized data from the annual national health survey, the KCHS, from 2008 to 2013 [2]. This dataset represents populations of community dwelling Korean adults aged 19 years and older. The data was collected in 253 community units consisting of 16 metropolitan cities and provinces [2]. The samples in the KCHS were extracted based on two stratified sampling procedures. In the first stage of sampling, the primary sampling units were selected according to the resident area (i.e., cities and type of households). In the second stage of sampling, the number of households was selected based on the size of the identified resident areas and the proportional composition of educational attainment of family members in the household. The sampling methods ensured that the samples of the KCHS would be representative of the entire Korean population. Trained surveyors visited the selected households and conducted face-to-face interviews using computer assisted personal interviewing.

The KCHS covers various health topics: basic demographics, health behaviors (smoking, drinking, and exercise), mental health, chronic diseases, quality of life, accidents (i.e., car accidents, falls, etc.), and nutrition. Since 2008, fall accidents and related information were collected in the survey (Table 1).

The $2012 \mathrm{KCHS}$ did not include the questions about falls, and, thus fall statistics in 2012 are not reported in this paper. The KCHS contained longitudinal descriptive statistics, such as the incidence rates of falling requiring medical treatment, reasons for falls, and locations of falls for at least 2 of the 5 consecutive years.

\section{Identification of fall accidents}

Fall accidents were not consistently defined across the 2008-2013 KCHSs. For the purpose of this study, we defined a fall accident as a fall that required medical treatment in the 12 months immediately following, and the definition of fall accidents was used for all analyses. The participants were asked a series of questions related to fall accidents (see Table 1), such as "Have you experienced at least 1 fall accident that required medical treat- 
Table 1. Korean Community Health Survey variables related to fall accidents 2008-2013

\begin{tabular}{|c|c|c|c|c|c|c|}
\hline Variable & 2008 & 2009 & 2010 & 2011 & $2012^{\text {a) }}$ & 2013 \\
\hline Demographics & o & o & o & o & - & o \\
\hline Chronic diseases & o & o & o & o & - & 0 \\
\hline Fall accidents & o & o & o & o & - & o \\
\hline Fall accidents requiring medical treatments & o & o & o & o & - & o \\
\hline Fall accidents not requiring medical treatments & - & - & - & o & - & 0 \\
\hline Number of falls & o & 0 & o & o & - & o \\
\hline Fall location & o & o & - & o & - & o \\
\hline Month of falls & 0 & o & o & - & - & - \\
\hline Reasons for the falls & - & - & - & o & - & o \\
\hline Fear of falling & - & - & - & - & - & o \\
\hline Activity when fall occurred & - & o & - & - & - & - \\
\hline
\end{tabular}

a) 2012 survey did not include questions regarding fall accidents; o, included; -, not included.

ments in the last 12 months? A fall accident may include slipping, tripping or stumbling." All subjects gave written informed consent before completing the KCHS, and the Institutional Review Board of the Korean Centers for Disease Control and Prevention (No. 2013-06EXP-01-3C) approved this study.

\section{Statistical analysis}

All test results were weighted to represent the Korean adult population. Descriptive statistics, such as frequencies and percentages, were used to describe the characteristics of data. Longitudinal incidence rates of fall accidents (per 100,000) requiring medical treatments were calculated from 2008 to 2013. The Cochran-Armitage trend test was used to examine the trends of fall accidents with 2 levels of categorical variables, including sex and fall location. Chi-square tests were used to test the trends of fall accidents with more than 2 levels of categorical variables, including age group and number of falls [12]. Pearson correlation was used to test the relationship between the trends of fall accident occurrence and the location proportions of these fall accidents for 7 years. We performed multivariable logistic regression to calculate the adjusted odds ratios (ORs) in order to identify the risk factors for fall accidents and fear of falls. In logistic regression models, we included covariates, including demographics and chronic conditions, which were identified from previous community-based studies $[4,5,11,13]$. Statistical significance was determined at the 0.05 level, and results were expressed with a $95 \%$ confidence interval (CI). All analyses were performed using SAS statistical software ver. 9.4 (SAS Institute Inc., Cary, NC, USA) for complex survey designs.

\section{RESULTS}

The sample sizes of the data were 220,258 in 2008, 230,715 in 2009, 229,229 in 2010, 229,226 in 2011, and 228,781 in 2013. Table 2 presents the descriptive characteristics of the study participants who experienced at least 1 fall accident that required medical treatment during the last 12 months in each year. Females had more fall accidents than males in each year $(\mathrm{p}<0.001)$. The proportion of females who experienced fall accidents gradually increased across the 5 -year datasets $(53.43 \%$ in 2008 to $58.24 \%$ in 2013; $\mathrm{p}<0.001$ ).

Fig. 1 presents the trends of fall accidents in age groups across the consecutive years. Fall accidents in adults between the ages of 18 and 44 years rapidly increased between 2010 and $2011(\mathrm{p}<0.001)$. Fall accidents in adults between the ages of 45 and 65 years old consistently decreased, except for 2010 ( $\mathrm{p}<0.001)$. Fall accidents in adults over 85 years old gradually decreased, except in 2009 $(\mathrm{p}<0.001)$. Fall accidents in adults between the ages of 65 and 84 years rapidly decreased between 2010 and 2011 $(\mathrm{p}<0.001$ ). Since 2008, the incidence rates (per 100,000 people) of falls increased significantly $(\mathrm{p}<0.001)$, and the incidence rate in 2013 (3,423 per 100,000 people) was 2.74 times of the incidence rate in 2008 ( 1,248 per 100,000 people). Fig. 2 presents the incidence rates among age groups across consecutive years. Old age groups demonstrated high incidence rates compared to young age 
Table 2. Characteristics of adults with fall accidents accompanying medical treatments 2008-2013

\begin{tabular}{|c|c|c|c|c|c|c|}
\hline Characteristic & 2008 & 2009 & 2010 & 2011 & 2013 & $p$ for trend \\
\hline Incidence rate ${ }^{\text {a) }}$ & 1,248 & 1,260 & 1,160 & 3,170 & 3,423 & $<0.001^{* * *}$ \\
\hline Age (yr) & & & & & & $<0.001^{* * *}$ \\
\hline $18-44$ & $33.15(157,431)$ & $36.80(178,692)$ & $34.14(154,662)$ & $42.38(536,077)$ & $41.29(576,380)$ & \\
\hline $45-64$ & $41.62(197,689)$ & $37.78(183,415)$ & $38.82(175,865)$ & $33.57(424,663)$ & $33.35(465,542)$ & \\
\hline $65-84$ & $23.29(110,635)$ & $23.83(115,679)$ & $25.21(114,170)$ & $22.42(283,568)$ & $23.93(334,033)$ & \\
\hline$\geq 85$ & $1.94(9,206)$ & $2.59(7,736)$ & $1.83(8,297)$ & $1.63(20,664)$ & $1.43(19,996)$ & \\
\hline Gender & & & & & & $<0.001^{* * *}$ \\
\hline Male & $44.57(211,673)$ & $46.18(224,199)$ & $42.01(190,308)$ & $43.37(548,625)$ & $41.76(582,964)$ & \\
\hline Female & $55.43(263,288)$ & $53.82(261,323)$ & $57.99(262,686)$ & $56.63(716,347)$ & $58.24(812,987)$ & \\
\hline Number of falls & & & & & & $<0.001^{* * *}$ \\
\hline 1 & $96.52(457,626)$ & $95.42(462,986)$ & $92.96(421,113)$ & $66.42(840,213)$ & $64.12(895,031)$ & \\
\hline 2 & $2.94(13,946)$ & $4.11(19,947)$ & $6.03(27,307)$ & $18.85(238,485)$ & $19.30(269,476)$ & \\
\hline 3 or more & $0.54(2,571)$ & $0.47(2,278)$ & $1.01(4,574)$ & $14.73(186,273)$ & $16.58(231,443)$ & \\
\hline Missing & 817 & 312 & 0 & 0 & 0 & \\
\hline Fall location & & & & & & $<0.001^{* * *}$ \\
\hline Indoor & $38.12(179,899)$ & $36.59(177,526)$ & NA & $23.07(291,683)$ & $23.16(323,290)$ & \\
\hline Outdoor & $61.88(292,002)$ & $63.41(307,629)$ & NA & $76.93(972,925)$ & $76.84(1,072,587)$ & \\
\hline Missing & 3,060 & 367 & NA & 363 & 74 & \\
\hline Total & 474,961 & 485,522 & 452,994 & $1,264,972$ & $1,395,951$ & \\
\hline
\end{tabular}

Values are presented as percentage (number) or number.

NA, not applicable.

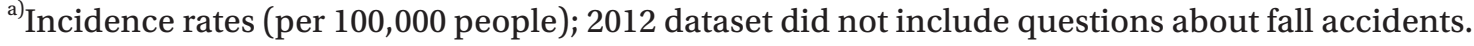

Cochran-Armitage trend test was used to examine the trends of fall accidents.

*** $\mathrm{p}<0.001$.

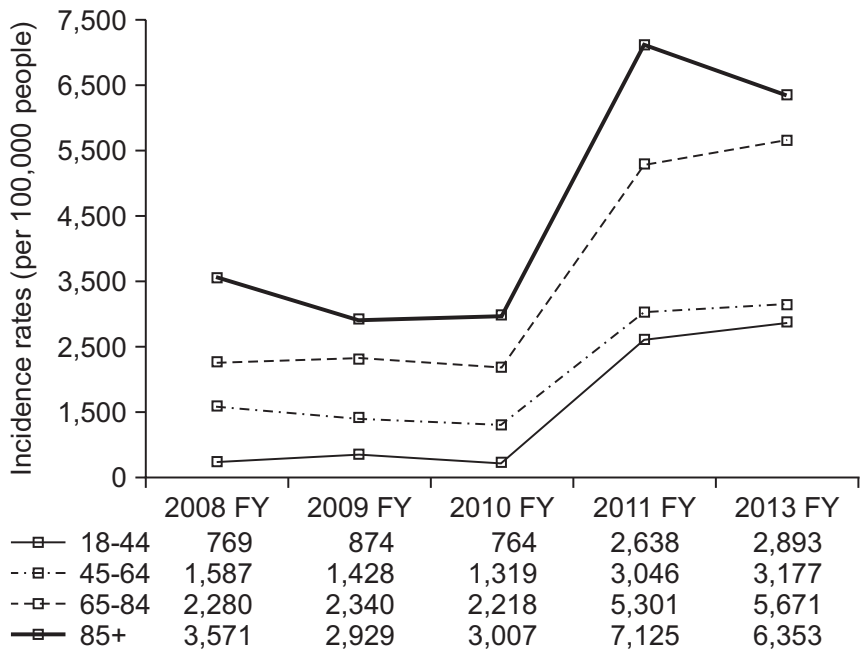

Fig. 1. Fall accidents in age groups across consecutive years, 2008 to 2013 .

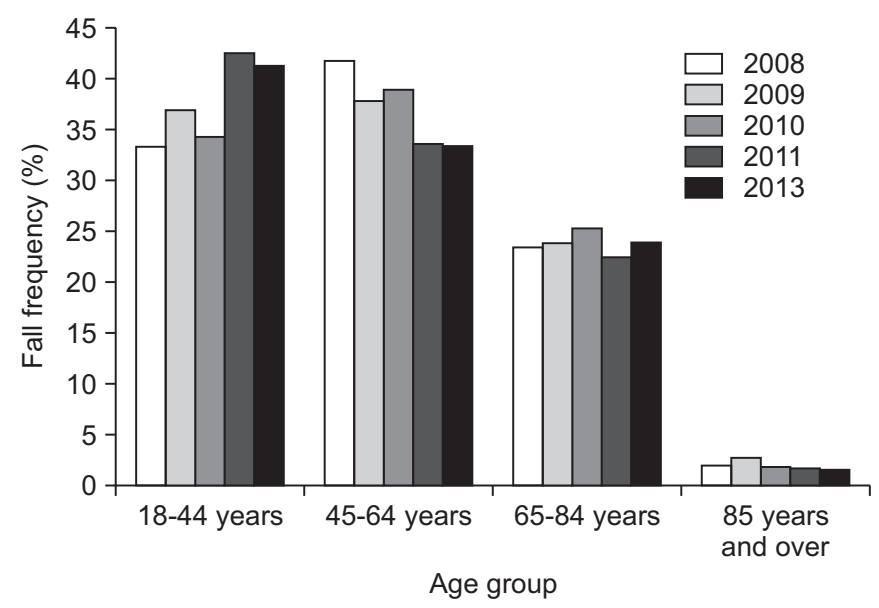

Fig. 2. Incidence rates among age groups across consecutive years, 2008 to 2013 .

groups, figures not shown. At the same time, the percentage of indoor fall accidents also consistently decreased from $31.12 \%$ in 2008 to $23.16 \%$ in 2013 ( $p<0.001)$. The 
correlation between the incidence rates and proportion of indoor fall accidents, and the correlation between the incidence rates and proportion of outdoor fall accidents in 2008, 2009, 2011, and 2013 showed strong relationships $(\mathrm{r}=-0.99, \mathrm{p}=0.007$ and $\mathrm{r}=0.99, \mathrm{p}=0.007$, respectively). Table 2 presents a significant increase in the number of individuals experiencing more than 2 fall accidents, from $4.09 \%$ in 2011 to $33.17 \%$ in 2013 ( $\mathrm{p}<0.001$ ). In 2009, people experienced fall accidents while performing basic daily activities $(55.58 \%)$, working $(21.28 \%)$, other activities $(7.29 \%)$, exercise $(7.33 \%)$, social activities $(4.50 \%)$, playing $(3.02 \%)$, and studying $(0.98 \%)$.

The major reason for fall accidents was slippery floors (33.7\% in 2011 and $36.3 \%$ in 2013), with additional reasons including the following: making a false step $(19.8 \%$ in 2011 and $19.1 \%$ in 2013), caught in a footpath or the threshold (11.1\% in 2011 and $12.4 \%$ in 2013), felt dizzy or falling unconscious (11.04\% in 2011 and $11.5 \%$ in 2013), hit by people or objects (10.4\% in 2011 and $9.7 \%$ in 2013), other people caused the fall $(9.7 \%$ in 2011 and $6.7 \%$ in 2013), steep slopes (3.5\% in 2011 and $3.2 \%$ in 2013), and poor lighting ( $0.9 \%$ in 2011 and $0.6 \%$ in 2013). Most fall accidents occurred in August and September in 2008, 2009, and 2010.

Fig. 3 presents the frequency of fall accidents across a 12-month period between 2008 and 2010. The overall fall accident frequency for the 3 consecutive years demonstrated similar trends. Fall accidents increased in July

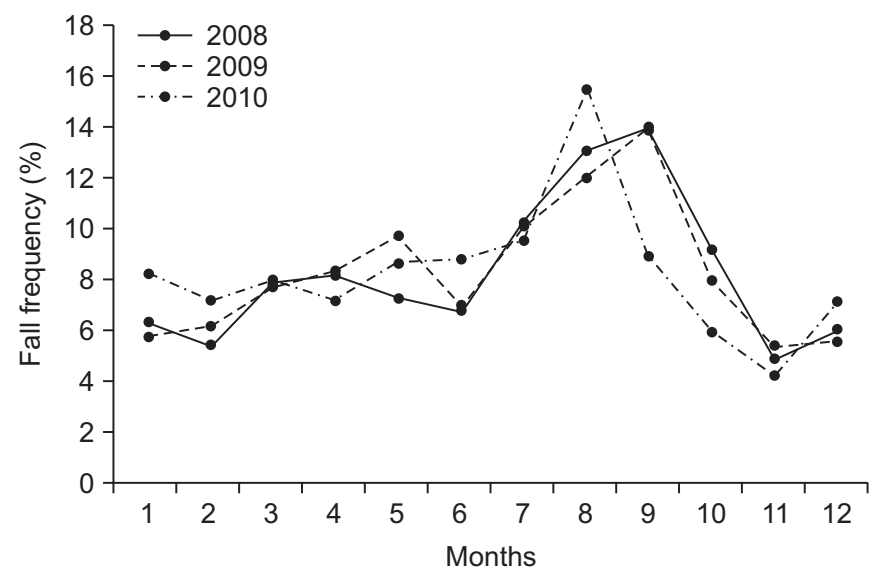

Fig. 3. Fall accident frequency across 12 months between 2008 and 2010. Most fall accidents occurred in August and September.

Table 3. Multivariable logistic regression analysis of risk factors for fall accidents in 2010

\begin{tabular}{|c|c|c|c|}
\hline Variable & $\mathbf{O R}^{\mathrm{a})}$ & $95 \% \mathrm{CI}$ & p-value \\
\hline August and September & 33.27 & 29.29-37.78 & $<0.001^{* * *}$ \\
\hline Age (per 10 years) & 1.14 & $1.09-1.20$ & $<0.001^{* * *}$ \\
\hline Female & 1.33 & $1.14-1.57$ & $<0.001^{* * *}$ \\
\hline Overweight (BMI $\geq 25$ vs. BMI <25) & 0.95 & $0.79-1.15$ & 0.61 \\
\hline Married (married/partnered vs. never married/non-partnered) & 0.85 & $0.76-0.95$ & $0.006^{* *}$ \\
\hline Education (higher than elementary school vs. uneducated) & 0.85 & $0.73-0.99$ & $0.04^{*}$ \\
\hline Household income ( $>\$ 1,000$ vs. $<\$ 1,000$ a month) & 1.05 & $0.91-1.02$ & 0.50 \\
\hline Residence type (attached vs. detached house) & 0.97 & $0.86-1.10$ & 0.53 \\
\hline Physical activity ( $\geq 2$ days vs. never a week) & 0.99 & $0.90-1.11$ & 0.97 \\
\hline Current alcohol intake & 1.20 & $1.10-1.35$ & $0.002^{* *}$ \\
\hline Current smoker & 1.32 & $1.14-1.54$ & $<0.001^{* * *}$ \\
\hline Diabetes & 1.22 & $1.00-1.47$ & $0.04^{*}$ \\
\hline Osteoarthritis & 1.24 & $1.04-1.46$ & $0.02 *$ \\
\hline Osteoporosis & 1.77 & $1.46-2.16$ & $<0.001^{* * *}$ \\
\hline Depression & 1.56 & $1.17-2.06$ & $0.002^{* *}$ \\
\hline Hypertension & 0.96 & $0.84-1.10$ & 0.56 \\
\hline Stroke & 1.44 & $1.00-2.07$ & 0.05 \\
\hline Cataracts & 1.11 & $0.91-1.35$ & 0.31 \\
\hline
\end{tabular}

OR, odds ratio; CI, confidence interval; BMI, body mass index $\left(\mathrm{kg} / \mathrm{m}^{2}\right)$.

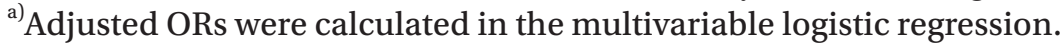

${ }^{*} \mathrm{p}<0.05 ;{ }^{* *} \mathrm{p}<0.01 ;{ }^{* * *} \mathrm{p}<0.001$. 
and reached the highest fall accident frequency in August and September. Fall accidents decreased in October and reached the lowest frequency in November.

Table 3 represents the multivariable logistic regression analysis of the risk of fall accidents in the most recent dataset of 2010. The following multivariable-adjusted ORs predicted a higher risk of fall accidents: in specific months (OR for August and September, 33.27; 95\% CI, 29.29-37.78), older age (OR per 10 years, 1.14; 95\% CI, 1.09-1.20), female gender (OR, 1.33; 95\% CI, 1.14-1.57), current alcohol intake (OR, 1.20; 95\% CI, 1.10-1.35), current smokers (OR, 1.32; 95\% CI, 1.14-1.54), and in people with diabetes (OR, 1.22; 95\% CI, 1.00-1.47), osteoarthritis (OR, 1.24; 95\% CI, 1.04-1.46), osteoporosis (OR, 1.77; 95\% CI, 1.46-2.16), and depression (OR, 1.56; 95\% CI, 1.172.06). Being married (OR, 0.85; 95\% CI, 0.76-0.95) and having a higher education (OR, 1.05; 95\% CI, 0.73-0.99) were associated with a lower risk of fall accidents while being overweight, household income levels, residence type, physical activity, stroke history, and cataract history were not associated with a risk of any fall accidents in the past 12 months. The interaction effects between the specific month (August and September) and the rest of variables was also investigated. The specific month demonstrated the interaction effects with age (OR per 10 years, 1.12; 95\% CI, 1.04-1.21), female gender (OR, 1.54; 95\% CI, 1.21-1.96) and physical activity (OR, 0.73; 95\% CI, 0.58-0.92). The risk factors of experiencing multiple fall accidents were higher in people with cataracts (OR, 2.75; 95\% CI, 1.37-5.52) and in current smokers (OR, 2.08; 95\% CI, 1.15-3.74) by more than 2 times; however, increased age (per 10 years) was associated with a low risk of multiple fall accidents compared to people between the ages of 18 and 29 years (OR, 0.63; 95\% CI, 0.52-0.77). Table 4 presents the multivariable logistic regression model for the risk of fear of falls in 2013. People who experienced 1 fall accident (OR, 2.12; 95\% CI, 2.04-2.12) and who had more than 2 fall accidents (OR, 2.97; 95\% CI, 2.83-3.10)

Table 4. Multivariable logistic regression analysis of risk factors for fear of falls in 2013

\begin{tabular}{|c|c|c|c|}
\hline Variable & $\mathbf{O R}^{\text {a) }}$ & 95\% CI & p-value \\
\hline \multicolumn{4}{|l|}{ Number of fall accidents } \\
\hline No fall & - & - & - \\
\hline 1 Fall vs. no fall & 2.12 & $2.04-2.12$ & $<0.001^{* * *}$ \\
\hline$\geq 2$ Falls vs. no fall & 2.97 & $2.83-3.10$ & $<0.001^{* * *}$ \\
\hline Age (per 10 years) & 1.35 & $1.34-1.37$ & $<0.001^{* * *}$ \\
\hline Female & 2.61 & $2.51-2.71$ & $<0.001^{* * *}$ \\
\hline Overweight (BMI $\geq 25$ vs. $\mathrm{BMI}<25$ ) & 0.80 & $0.76-0.84$ & $<0.001^{* * *}$ \\
\hline Married (married/partnered vs. never married/non-partnered) & 0.80 & $0.78-0.82$ & $<0.001^{* * *}$ \\
\hline Education (higher than elementary school vs. uneducated) & 0.76 & $0.73-0.79$ & $<0.001^{* * *}$ \\
\hline Household income ( $>\$ 1,000$ vs. $<\$ 1,000$ a month) & 0.78 & $0.75-0.80$ & $<0.001^{* * *}$ \\
\hline Residence type (attached vs. detached house) & 1.10 & $1.07-1.13$ & $<0.001^{* * *}$ \\
\hline Physical activity ( $\geq 2$ days vs. never a week) & 0.96 & $0.93-0.98$ & $<0.001^{* * *}$ \\
\hline Current alcohol intake & 0.93 & $0.90-0.95$ & $<0.001^{* * *}$ \\
\hline Current smoker & 0.93 & $0.89-0.97$ & $<0.001^{* * *}$ \\
\hline Diabetes & 1.21 & $1.15-1.27$ & $<0.001^{* * *}$ \\
\hline Osteoarthritis & 1.60 & $1.53-1.67$ & $<0.001^{* * *}$ \\
\hline Osteoporosis & 1.40 & $1.32-1.48$ & $<0.001^{* * *}$ \\
\hline Depression & 1.46 & $1.37-1.61$ & $<0.001^{* * *}$ \\
\hline Hypertension & 1.19 & $1.15-1.23$ & $<0.001^{* * *}$ \\
\hline Stroke & 1.93 & $1.72-2.17$ & $<0.001^{* * *}$ \\
\hline Cataracts & 1.37 & $1.30-1.44$ & $<0.001^{* * *}$ \\
\hline
\end{tabular}

OR, odds ratio; CI, confidence interval; BMI, body mass index $\left(\mathrm{kg} / \mathrm{m}^{2}\right)$; fear of falls 0 , never; 1 , some or lots of fear.

${ }^{a}$ Adjusted ORs were calculated in the multivariable logistic regression.

${ }^{*} \mathrm{p}<0.05 ;{ }^{* *} \mathrm{p}<0.01 ;{ }^{* * *} \mathrm{p}<0.001$. 
felt more fear of falls than those who had no fall accident experience within the last 12 months. Older adults, females, attached house dwellers (apartment or townhouse), or a presence of diabetes, osteoarthritis, osteoporosis, depression, hypertension, stroke, and cataracts were also associated with a higher risk of fear of falls. In contrast, people who were overweight, married, highly educated, wealthier, physically active, current alcohol intake, and current smokers had a lower risk of fear of falling.

\section{DISCUSSION}

We have identified the trends regarding fall accidents in Korean adults between 2008 and 2013 and found that fall accidents requiring medical treatments had increased over time. Adult females between 64 and 85 years old were more likely to experience fall accidents. The number of outdoor fall accidents and the number of adults having more than 2 fall accidents have increased over time. Variables associated with fall accident risks included specific months (August and September), age (10 years), female gender, current alcohol intake, current smoker, diabetes, osteoarthritis, osteoporosis, and depression. The major reason for fall accidents was slippery floors, and when people had fall accidents, they understandably expressed more fear of falling than those who had had no fall accidents.

To our knowledge, this study is the first to investigate the trends of fall accidents using a nationally representative sample of Korean adults for consecutive years. Although a previous study investigated risk factors of fall accidents using a national level dataset, the results were limited to a specific year and a population aged 65 years or older [11]. On the other hand, by analyzing the trends of fall accidents in various demographics and environmental characteristics for a consecutive 3-year period, we identified that seasonal changes, especially in August and September, was the major factor for fall accidents $(25.2 \%$ of falls). The seasonal change was previously an unknown risk factor for fall accidents, in contrast with age, which was the major risk factor of fall accidents identified by previous Korean and Western studies $[11,14]$. The seasonal change demonstrated interaction effects with age (OR, 1.12; 95\% CI, 1.04-1.21), female (OR, 1.54; 95\% CI, 1.21-1.96), and physical activity (OR, 0.73; 95\% CI,
0.58-0.92) indicating that adults over 30 years old experienced (per 10 years) $12 \%$ increased odds of fall accidents and females have a greater than $20 \%$ increased risk of fall accidents in August and September in 2010.

We were not able to test the interaction effects between the seasonal change and outdoor fall accidents with logistic regression models because the 2010 dataset did not contain the outdoor fall accident variable and there were considerable missing data in the outdoor fall accident variable in other available datasets, such as $96 \%$ missing data in 2008 and $95 \%$ missing data in 2009. Instead, we used chi-square tests for the interaction effects between seasonal change and the outdoor fall accident variable using the 2008 and 2009 dataset. In both datasets, there were significant relationships between outdoor fall accidents and seasonal change $(\mathrm{p}<0.001)$. However, as the results were not adjusted for other demographics and covariates, further studies are needed to test the interaction effects between outdoor fall accidents and seasonal change.

During these same months, among people who reported being regularly physically active (more than 2 days), the risk of falling was $27 \%$ lower compared to those who were never physically active. However, the fall accident frequencies across month are not consistent with previous studies [8,15]. In our study, most adults over 65 years old had fall accidents in August (16.6\%). A prospective study reported that older adults frequently had falls in April (12.8\%) and December (11.7\%) [8]. A communitybased study found that $38.9 \%$ of falls occurred between March and May [15]. Although the differences could be influenced by recall bias, a particular sub-population, and loss of memory in older adults, our findings were consistent for 3 consecutive years from 2008 to 2010 .

The increasing fall accidents in Korea may be explained by environmental factors, such as lifestyle changes across the nation. Korea has experienced remarkable socioeconomic growth that has resulted in significant changes in nutrition, leisure, and lifestyle [16]. For example, $48 \%$ of Koreans spent more than 3 hours of leisure time on weekends in 2011, and $61 \%$ of people reported spending the same amount of leisure time in 2013 [17]. In 2012, Koreans enjoyed more outdoor leisure activities (58.0\%) than indoor activities (41.9\%) [18]. Our findings also suggested that the lifestyle changes are associated with increasing fall accidents $(r=0.99, p=0.007)$, and the propor- 
tion of outdoor falls has increased from $68.9 \%$ in 2008 to $76.8 \%$ in 2013.

Our finding that females had more fall accidents than males is consistent with previous Korean and Western studies $[4,11,14,15]$. The differences in muscle strength levels may contribute to the high rate of falls in women. The loss of lower body strength is strongly associated with increasing risk of fall accidents $[19,20]$. Previous Western studies have suggested that men have greater lower body strength than women [19-21] and, thus, males were at a relatively lower risk of falls compared to women. Unfortunately, the KCHS is based on interviews and does not include physiological measures, such as muscle strength, difference in gate, or knee action; therefore, we were not able to test the gender difference in relation to physiological functions. A possible explanation is that a high risk of falls in women was associated with osteoporosis [22]. Our finding supports the fact that women were at a higher risk of osteoporosis than men (OR, 8.28; 95\% CI, 7.33-9.36). However, future research is needed to clarify the relationship between gender difference and risk of fall accidents. Between 2008 and 2013, the proportion of people experiencing more than 2 fall accidents in a 12 -month period has consistently increased, and people suffering more than 2 fall accidents felt more fear of falling than nonfallers (OR, 2.97; 95\% CI, 2.83-3.10). A previous study suggested that $46 \%$ of healthy older people were afraid of falling, and $56 \%$ of them curtailed daily activities due to their fear [23]. Reduced physical capacity can also increase the risk of falls $[19,20]$. Thus, with the trend of multiple fall accidents increasing along with their fear of falling, community dwelling Korean people are expected to be at a greater risk of potential falls than previously.

Various fall prevention programs showed their effectiveness at preventing fall accidents, such as multiplecomponent group exercise, assessment and multifactorial intervention, and home modification [24]. Also, various fall prevention programs and interventions have been established in Korea [25]. Despite these efforts, the fall incidence rate has increased since 2008. Fall accidents can cause severe injuries (or even death), as well as causing an increased social burden [1]. As the Korean population ages, fall accidents are expected to increase. Due to the multifactorial characteristics of effective fall prevention programs (i.e., general medical assessment focusing on fall risks, fall history, medication intake, and the use of assistive devices) [26], individuals may not benefit from a fall prevention program without comprehensive social support. Thus, Korean society may need to establish a systematic social support system to prevent fall accidents.

This study has several limitations. Firstly, questions from each dataset concerning fall accidents and related information were not consistent each year, except for the number of fall accidents. For example, between 2008 and 2010, falls were defined as having at leastl fall that required seeking medical treatment in the past year; however, since 2011, the KCHS has asked more comprehensive questions about fall accidents, both those requiring and not requiring medical treatment. In addition, some questions, such as reasons for falls, location of falls, fear of falling, and activities when falls occurred, were limited in a specific year. For these reasons, we were not able to test their trends. Therefore, the KCHS should include consistent questions about fall accidents in order to track stable trends. Secondly, the high increase in incidence rates between 2010 and 2011 could be a function of inconsistent questioning concerning fall accidents in the KCHS, such as the model of questioning regarding fall accidents. Therefore, the incidence rates after 2011 and 2013 could be inflated, compared to the incidence rates in 2008, 2009, and 2010. Finally, the KCHS does not include people in medical hospitals, skilled nursing facilities, and military facilities, so the results of similar analysis among those groups may differ.

\section{CONFLICT OF INTEREST}

No potential conflict of interest relevant to this article was reported.

\section{ACKNOWLEDGMENTS}

We would like to show our gratitude to Dr. Kit N. Simpson and Dr. Craig A. Velozo for providing their research ideas and statistical consultations. The Korean Centers for Disease Control and Prevention (KCDC) supported the study and provided the study data. We retrieved the study data from the KCDC webpage (chs.cdc.go.kr).

\section{REFERENCES}

1. Statistics Korea. Annual report on the cause of death statistics. Daejeon: Statistics Korea; 2014. 
2. Kim YT, Choi BY, Lee KO, Kim H, Chun JH, Kim SY, et al. Overview of Korean community health survey. J Korean Med Assoc 2012; 55:74-83.

3. Cho JP, Paek KW, Song HJ, Jung YS, Moon HW. Prevalence and associated factors of falls in the elderly community. Korean J Prev Med 2001;34:47-54.

4. Lim NG, Shim KB, Kim YB, Park JL, Kim EY, Na BJ, et al. A study on the prevalence and associated factors of falls in some rural elderly. J Korean Geriatr Soc 2002;6:183-96.

5. Shin KR, Kang Y, Hwang EH, Jung D. The prevalence, characteristics and correlates of falls in Korean community-dwelling older adults. Int Nurs Rev 2009;56: 387-92.

6. Tinetti ME, Speechley M, Ginter SF. Risk factors for falls among elderly persons living in the community. N Engl J Med 1988;319:1701-7.

7. Colling J, Park D. Home, safe home. J Gerontol Nurs 1983;9:175-9.

8. Lim JY, Park WB, Oh MK, Kang EK, Paik NJ. Falls in a proportional region population in Korean elderly: incidence, consequences, and risk factors. J Korean Geriatr Soc 2010;14:8-17.

9. Korea Centers for Disease Control and Prevention. 2014 Korean Community Health Survey. Cheongju: Centers for Disease Control and Prevention; 2014.

10. Korea Centers for Disease Control and Prevention. Having a quick look at the regional statistics between 2008 and 2011. Cheongju: Centers for Disease Control and Prevention; 2012.

11. Choi EJ, Kim SA, Kim NR, Rhee JA, Yun YW, Shin MH. Risk factors for falls in older Korean adults: the 2011 Community Health Survey. J Korean Med Sci 2014;29:1482-7.

12. Armitage P. Tests for linear trends in proportions and frequencies. Biometrics 1955;11:375-86.

13. Kim JM, Lee MS, Song HJ. An analysis of risk factors for falls in the elderly by gender. Korean J Health Educ Promot 2008;25:1-18.

14. Deandrea S, Lucenteforte E, Bravi F, Foschi R, La Vecchia C, Negri E. Risk factors for falls in communitydwelling older people: a systematic review and metaanalysis. Epidemiology 2010;21:658-68.

15. Kim JM, Lee MS. Risk factors for falls in the elderly population in Korea: an analysis of the third Korea
National Health and Nutrition Examination Survey data. Korean J Health Educ Promot 2007;24:23-39.

16. Han MA, Kim KS, Park J, Kang MG, Ryu SY. Association between levels of physical activity and poor self-rated health in Korean adults: the Third Korea National Health and Nutrition Examination Survey (KNHANES), 2005. Public Health 2009;123:665-9.

17. Korea Ministry of Culture Sports and Tourism. Survey on citizen's sport participation 2013. Sejong: Ministry of Culture Sports and Tourism; 2013.

18. Korea Ministry of Culture Sports and Tourism. Survey on citizen's leisure participation 2012. Sejong: Ministry of Culture Sports and Tourism; 2012.

19. Stevens JA, Sogolow ED. Gender differences for nonfatal unintentional fall related injuries among older adults. Inj Prev 2005;11:115-9.

20. American Geriatrics Society; British Geriatrics Society; American Academy of Orthopaedic Surgeons Panel On Falls Prevention. Guideline for the prevention of falls in older persons.

21. de Rekeneire N, Visser M, Peila R, Nevitt MC, Cauley JA, Tylavsky FA, et al. Is a fall just a fall: correlates of falling in healthy older persons. The Health, Aging and Body Composition Study. J Am Geriatr Soc 2003;51:841-6.

22. Fletcher PC, Hirdes JP. Risk factors for falling among community-based seniors using home care services. J Gerontol A Biol Sci Med Sci 2002;57:M504-10.

23. Howland J, Peterson EW, Levin WC, Fried L, Pordon D, Bak S. Fear of falling among the community-dwelling elderly. J Aging Health 1993;5:229-43.

24. Gillespie LD, Robertson MC, Gillespie WJ, Lamb SE, Gates S, Cumming RG, et al. Interventions for preventing falls in older people living in the community. Cochrane Database Syst Rev 2009;2:CD007146.

25. Sohng KY, Moon JS, Song HH, Lee KS, Kim YS. Fall prevention exercise program for fall risk factor reduction of the community-dwelling elderly in Korea. Yonsei Med J 2003;44:883-91.

26. Neyens JC, Dijcks BP, van Haastregt JC, de Witte LP, van den Heuvel WJ, Crebolder HF, et al. The development of a multidisciplinary fall risk evaluation tool for demented nursing home patients in the Netherlands. BMC Public Health 2006;6:74. 